\title{
Ecohydrologie urbaine et changement climatique
}

\author{
Pascal Breil ${ }^{1}$, Abdoulaye Faty ${ }^{2}$, and Didier Orange ${ }^{3}$ \\ ${ }^{1}$ INRAE, RiverLy, 5 Rue de la Doua, CS 20244, 69625, Villeurbanne, France \\ ${ }^{2}$ UCAD, Université Cheikh Anta Diop, Faculté de Géographie, UMI RESILIENCES, 10700, Dakar, Sénégal \\ ${ }^{3}$ IRD Institut de Recherche pour le Développement UMR Unité Mixte de Recherche, Université Montpellier, \\ CIRAD, INRAE, Institut SupAgro, UMR Eco\&Sols, LMI IESOL, 18524, Dakar, Sénégal
}

Correspondence: Pascal Breil (pascal.breil@inrae.fr)

Published: 16 November 2021

\begin{abstract}
Résumé. Due to global change, cities of the future will have to deal with more intense runoff and longer drought sequences, in addition to a growing urban and peri-urban population. French Mediterranean cities, such as Toulon, are already densely urbanised and exposed to the effects of global warming. The adaptation of their infrastructures is problematic. Cities with high development potential, such as Dakar, offer the opportunity to imagine other solutions for the management of water resources and its extremes in the context of global change. In particular, it is a question of managing the flows of water and substances linked to intense runoff events according to an ecohydrological logic that makes it possible to reduce environmental risks and increase social and economic benefits. To do this, we use a hydrologically-based geomatics model (IRIP) that produces predictive maps of areas of generation, transfer and accumulation of intense runoff and associated nutrients. This allows us to target effective intervention areas to reduce risks and increase water resources, for example by simulating land use change in appropriate locations and at the same time stimulating specific biological processes. The fundamental principle of ecohydrology is to balance energy flows with biological metabolic flows at the subcatchment scale. The mapping of intense runoff processes is a first step illustrated in this article for the cities of Toulon and Dakar. This first step is part of the Dakar'2030 project, which aims to rethink urban development and adapt it to climate change.
\end{abstract}

\section{Introduction}

Pour le groupe d'experts méditerranéens sur le changement climatique et environnemental (Cramer et al., 2019), la région méditerranéenne présente une augmentation de sa température annuelle moyenne supérieure $\left(+1,5^{\circ} \mathrm{C}\right)$ à celle observée au niveau mondial $\left(+1,1^{\circ} \mathrm{C}\right)$. Les conséquences de ce réchauffement climatique «accéléré » en Méditerranée sont nombreuses, dont celles en lien avec le ruissellement intense pluvial. Ainsi, des sécheresses plus longues et des pluies torrentielles pourraient causer des problèmes majeurs pour l'agriculture, avec des pertes de production imprévues du fait de l'érosion des sols et de la perte en matière organique. En corolaire, les besoins en irrigation devraient augmenter ainsi que les incendies. Les zones humides, réservoirs de biodiversité et régulateurs des parasites seraient fortement menacées. Le risque d'inondation par ruissellement et de destruction par coulées de boue augmenterait aussi. Les inondations urbaines favoriseraient la transmission des maladies. Face à ces défis la gestion des espaces pour ralentir et conserver l'eau et les sols est une solution à envisager. L'écohydrologie propose le principe de la régulation duale (Zalewski, 2014) : les flux d'eau transfèrent les flux d'énergie associés (substances dissoutes et particulaires, thermie) vers les systèmes biotiques (microbes, plantes, chaine trophique) qui les transforment sous forme de biomasse ou de réactions biochimiques. Les flux d'eaux sont ainsi modifiés en quantité et en qualité par les interactions entre les systèmes biotiques et abiotiques. Si l'on souhaite réguler ces flux à l'échelle d'un bassin versant pour limiter les effets décrits du changement climatique, et promouvoir les services écosystémiques, il est nécessaire de représenter les circulations d'eau, en particulier lors des conditions de pluies intenses pour deux raisons : (I) localiser les pertes en sol, les destructions de cultures et d'in- 
frastructures, les inondations ou encore des dépôts de matières en zone rurale ou urbaine; (II) localiser les redistributions des nutriments produits et accumulés par les processus naturels ou par l'homme en temps normal. L'objectif est de minimiser les risques tout en utilisant les forces hydrauliques naturelles pour orienter les transferts de nutriments qui soutiennent les services écosystémiques.

\subsection{Pluies plus intenses, sécheresses et réseaux d'assainissement}

Parmi les risques dérivés, les processus d'érosion des sols par l'effet des sécheresses comme des pluies intenses sont de nature à créer de nombreux dysfonctionnements des réseaux d'assainissement unitaires et d'eau pluviales. En ce sens l'expérience africaine en climat tropical avec une longue saison sèche comme à Dakar peut s'avérer riche d'enseignement pour anticiper des problèmes de gestion du pourtour méditerranéen. En particulier, il a souvent été noté que la construction de réseaux d'assainissement unitaire impliquerait à Dakar un surdimensionnement pour absorber le ruissellement des pluies tropicales. Par ailleurs les longues périodes sèches sont néfastes au bon fonctionnement d'un réseau qui aura tendance à s'encombrer faute de capacité d'auto-curage, renforcé par des dimensions importantes et par l'apport d'eau ruisselée très chargée en flottants et sédiments divers lors des pluies orageuses. Les nuisances sont olfactives et sanitaires par débordement ou refus des réseaux. Enfin, le développement urbain sur plusieurs décennies se traduit systématiquement par un dépassement des capacités des réseaux anciens situés profondément dans les centres historiques des villes. Ce sont des goulots d'étranglement qu'il est difficile d'adapter aux flux gravitaires en provenance des zones périurbaines qui s'étendent avec l'accroissement urbain. Les raisons historiques, sociologiques et économiques de cette situation sont connues et posent aujourd'hui un problème majeur d'adaptation pour les gestionnaires des pays industriels. En effet, le coût de la réhabilitation des réseaux anciens qui se dégradent en vieillissant peut être insurmontable. Et le changement climatique n'est pas de nature à améliorer la situation car les pluies intenses ne pourront être gérées par des réseaux déjà défaillants.

\subsection{La gestion alternative au « tout au tuyau » ... mais pas seulement en zone urbaine}

Une stratégie de gestion alternative au tout tuyau pour les zones urbaines semble dès lors s'imposer pour gérer au mieux les flux d'eau, de sédiment et de substances transportés par le ruissellement. La gestion dite «à la source» consiste à stocker temporairement ou à infiltrer l'eau de ruissellement à proximité de sa zone de production. Cette possibilité est cependant contrainte par la disponibilité de l'espace en zone urbaine dense. Et il s'agit toujours dans ces solutions de gérer l'auto-inondation urbaine qui résulte du ruisselle- ment généré par la ville elle-même. Dans le cas du changement climatique, les pluies plus intenses vont étendre les aires de connexions entre les zones périurbaines de l'amont et les centres urbains. Dans ce tissu rural et urbain non encore fortement imperméabilisé, le ruissellement intense peut résulter d'une intensité de pluie supérieure à la vitesse d'infiltration dans le sol ou encore par effet de saturation du profil de sol suite à des pluies répétées qui laissent peu de temps au ressuyage naturel. Les zones périurbaines sont donc stratégiques pour réguler les flux de ruissellement dans une logique écohydrologique qui permet de réduire les risques et d'accroitre les bénéfices. Le recyclage des eaux usées peut être intégré à cette logique d'ensemble pour ne pas surcharger les réseaux d'assainissement en usage. Cela nécessite cependant de mettre en place une planification de l'usage des sols entre naturel, agricole, urbain mais aussi dédiés à l'éco-ingénierie. Une première étape consiste à comprendre comment les processus du ruissellement intense s'organisent dans l'espace d'un bassin versant. La régulation duale des flux est regardée sur des exemples locaux tirés des cartographies du ruissellement intense produites par un modèle. La représentation cartographique des effets du ruissellement permet de préciser où des actions de régulation des flux d'eau et d'énergie seraient les plus efficaces pour fournir des services écosystémiques et réduire des risques potentiels. Dans les axes de transfert il est possible de casser l'énergie pour réduire l'érosion mécanique et les pertes en sol. Dans les zones d'accumulation, il possible de développer de la biomasse végétale à haut rendement en recyclant les eaux pluviales et usées via des biofiltres naturels. Ainsi, la connaissance des lieux de production du ruissellement, de ses axes de transfert, et des lieux de son accumulation sont des informations importantes pour imaginer les stratégies de gestion à mettre en place. Aujourd'hui, le besoin de connaissance concerne des dizaines de kilomètres carrés en devenir d'urbanisation en périphérie des villes. Cette étude explore la faisabilité d'une telle gestion dans un cas d'urbanisation très dense comme à Toulon (France), et dans le cas d'une ville à fort potentiel de développement, comme à Dakar (Sénégal).

\section{Méthode}

Nous proposons à cette fin d'utiliser un modèle géomatique à base hydrologique pour décrire les zones de production $(P)$ du ruissellement intense, les zones de transfert $(T)$ et érosion liées aux vitesses d'écoulement et les zones d'accumulation $(A)$ d'eaux et de matières. Ce modèle, siglé IRIP pour Indicateurs du Ruissellement Intense Pluvial, a été décrit dans plusieurs publications (Lagadec et al., 2018; Braud et al., 2020) et fait l'objet d'évaluation de terrain pour en valider les facteurs. Le modèle IRIP est appliqué ci-après au bassin versant principal de la ville de Toulon et au territoire étendu de la péninsule de Dakar. Les scores varient de 0 à 
5 pour chacun des processus $P, T$ et $A$. Ils sont calculés en chacun des pixels définis par le modèle numérique de terrain.

\subsection{Les couches d'information en entrée du modèle IRIP}

Le modèle IRIP utilise 3 données de base : le modèle numérique de terrain (MNT) qui permet d'extraire le plan de drainage des écoulements de surface, mais aussi les mise en vitesse et les ralentissements selon les courbures topographiques concaves ou convexes; l'occupation du sol dont les caractéristiques sont traduites en facteurs favorables ou non au ruissellement, en particulier les couverts imperméables; et les caractéristiques pédologiques comme la texture, la densité apparente, dont sont dérivées des propriétés hydrodynamiques, la battance et l'érodibilité des sols. Les sources des données sont nationales (ISRIC data hub, 2020) et internationales dans les deux cas d'étude (voir Tableau 1).

Pour calculer la sensibilité des sols à produire du ruissellement hortonien, il a été considéré la pluie d'une heure de période de retour 5 ans pour Dakar (Bodian et al., 2016) et de 10 ans pour Toulon car elles sont comparables. Pour le ruissellement par saturation il est considéré la pluie de $24 \mathrm{~h}$ pour les mêmes périodes de retour.

\section{Résultats}

\subsection{La péninsule de Dakar}

La vue d'ensemble des cartes IRIP (Fig. 1a) avec les scores les plus forts $(3,4,5)$ montre la contribution des zones urbanisées à produire du ruissellement intense (en rouge).

Certains sols à nu sont aussi des zones de production. Les zones d'accumulation du ruissellement sont bien visibles sous la forme de tâches bleues dans le tissu urbain. On retrouve aussi la grande zone humide qui collecte le ruissellement des alentours. La Fig. 1b est un agrandissement sur un quartier situé près de la zone humide (voir localisation sur Fig. 1a). Les zones de production et de transfert sont présentes sur la périphérie. La courbe de niveau d'altitude $z=12 \mathrm{~m}$ est marquée en blanc. Elle montre qu'il est possible de réduire l'accumulation en la transférant par gravité à l'aide d'un drain (flèche blanche) en direction des terrasses agricoles situées en bordure de la zone humide. Cela permet de rediriger les nutriments vers des zones de production agricole.

La Fig. 1c est une image aérienne localisée sur la Fig. 1b. Elle montre des cultures réalisées sur les pentes qui plongent vers la zone humide. C'est sur ces pentes qu'il est stratégique d'aménager des cultures en terrasse (ce qui n'est pas le cas pour l'instant) pour capter le ruissellement pluvial et les flux de substances associés, cela afin de maintenir l'humidité des sols, d'accroitre la fertilité et la production agricole. Les cultures devraient être généralisées sur ces pentes encore peu exploitées, ce qui favorise actuellement l'érosion et la
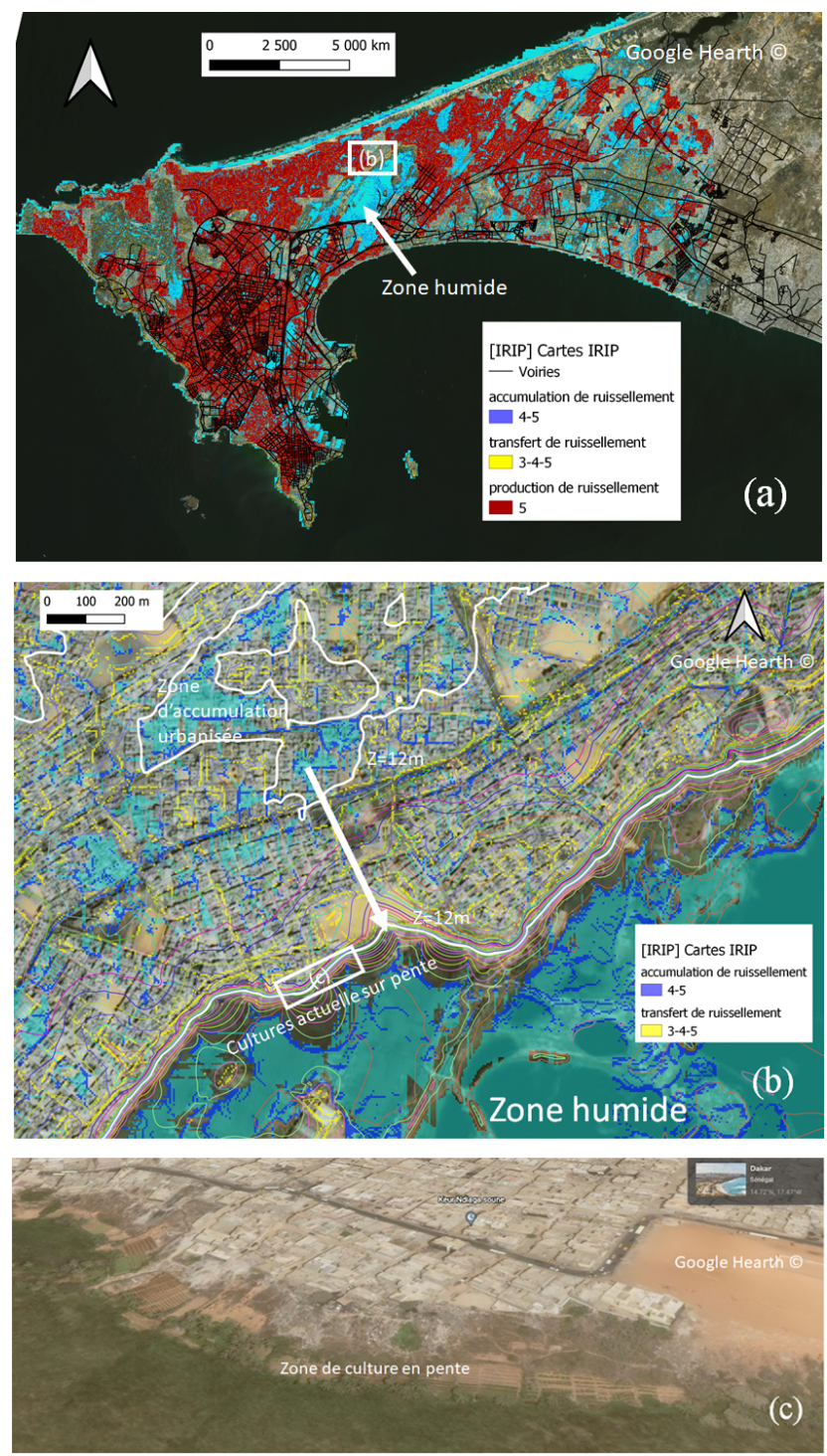

Figure 1. (a, b, c) Localisation des zones de production, transfertérosion et dépôt-inondation dans la péninsule de Dakar.

perte des sols. Elles devraient être aménagées en terrasses, comme les «fanya juu » très pratiqués en Afrique de l'Est. Les eaux domestiques peuvent alimenter ces cultures après passage dans les terrasses supérieures dédiées à leur autoépuration. Cela maintiendrait l'humidité et le flux de nutriments.

\subsection{Le bassin versant de la baie de Toulon}

Dans la Fig. 2a, il est noté la présence de massifs montagneux forestiers qui expliquent l'absence de ruissellement intense au nord-ouest et en partie au sud-est du bassin versant. Cela car les sols forestiers favorisent l'infiltration et que la présence des arbres réduits considérablement les processus érosifs. Le reste du bassin descend progressivement en plaine continue vers la mer Méditerranée. Le développement 
Tableau 1. Caractéristiques des données utilisées dans la modélisation IRIP.

\begin{tabular}{lll|ll}
\hline & \multicolumn{2}{c}{ Dakar } & \multicolumn{2}{c}{ Toulon } \\
\cline { 2 - 5 } & Résolution & Source & Résolution & Source \\
\hline Modèle numérique de terrain & $5 \mathrm{~m}$ & SRTM & $5 \mathrm{~m}$ & IGN \\
Occupation du sol & $250 \mathrm{~m}$ & ISRIC & $20 \mathrm{~m}$ & THEIA \\
Nature du sol & $250 \mathrm{~m}$ & Basegeo & $250 \mathrm{~m}$ & ESDAC \\
Pluie & régionale & Bodian et al. (2016) & régionale & Météo-France \\
Occurrence \& durée de pluie & 5 ans, 1 h & 5 ans, 24 h & 10 ans, $1 \mathrm{~h}$ & 10 ans, 24 h \\
Pluie mm & 45.0 & 64.8 & 48.5 & 118.1 \\
\hline
\end{tabular}

urbain est dense à Toulon et dans l'habitat périurbain. Deux zones humides d'importance sont visibles (aplats bleu). La plus grande a un rôle majeur de protection de la ville de Toulon contre les inondations. Elle est aussi très appréciée et fréquentée par les citadins pour son rôle d'ilot de fraicheur en période chaude. Elle améliore la qualité de l'eau des cours d'eau urbains qui l'alimente, avant de se déverser dans la baie de Toulon où l'activité économique est basée principalement sur le tourisme balnéaire.

La qualité des eaux de baignade est donc une contrainte forte. La grande zone humide fournit donc des services importants sur le plan économique et sanitaire. Il ne faut pas négliger son rôle de réservoir de biodiversité qui permet l'autorégulation des espèces, dont celles nuisibles aux cultures par exemple. Des zones de transfert érosif (jaune) sont présentes à flanc de massifs et en proximité de zones de très forte production (rouge) de ruissellement. Ces positions amont des aléas forts de ruissellement intense sont des facteurs de risque pour les habitations situées juste en aval. La pression urbaine est cependant très forte et des solutions d'aménagement contraintes sont à imaginer dans les secteurs exposés. La Fig. $2 \mathrm{~b}$ montre comment il est possible de protéger des habitations situées dans une zone d'accumulation à l'aide d'un bourrelet de terre érigé le long d'une courbe de niveau pour créer une zone de stockage transitoire du ruissellement urbain provenant de l'amont. La zone aval, qui est alors protégée, est figurée en jaune. La cartographie IRIP sert ici à identifier des situations critiques vis-à-vis des inondations pluviales et des coulées boueuses. La zone de stockage transitoire peut être conçue comme une mini zone humide, cela afin de favoriser les différents services écosystémiques déjà évoqués. Cela implique une étude d'éco-ingénierie prenant en compte les fréquences des apports d'eaux de ruissellement et leurs natures.

La Fig. 2c montre un système de terrasses agricoles appelées « restanques» qui apparait comme des zones d'accumulation fortes du ruissellement intense. Ces terrasses sont limitées par des lignes de transfert fort du ruissellement. Ces lignes correspondent aux murs de pierres sèches, non érodibles, qui soutiennent les terrasses. Le rôle des terrasses agricoles est ainsi bien restitué par la modélisation IRIP, ce qui autorise a priori la construction de scénarios d'aménage-
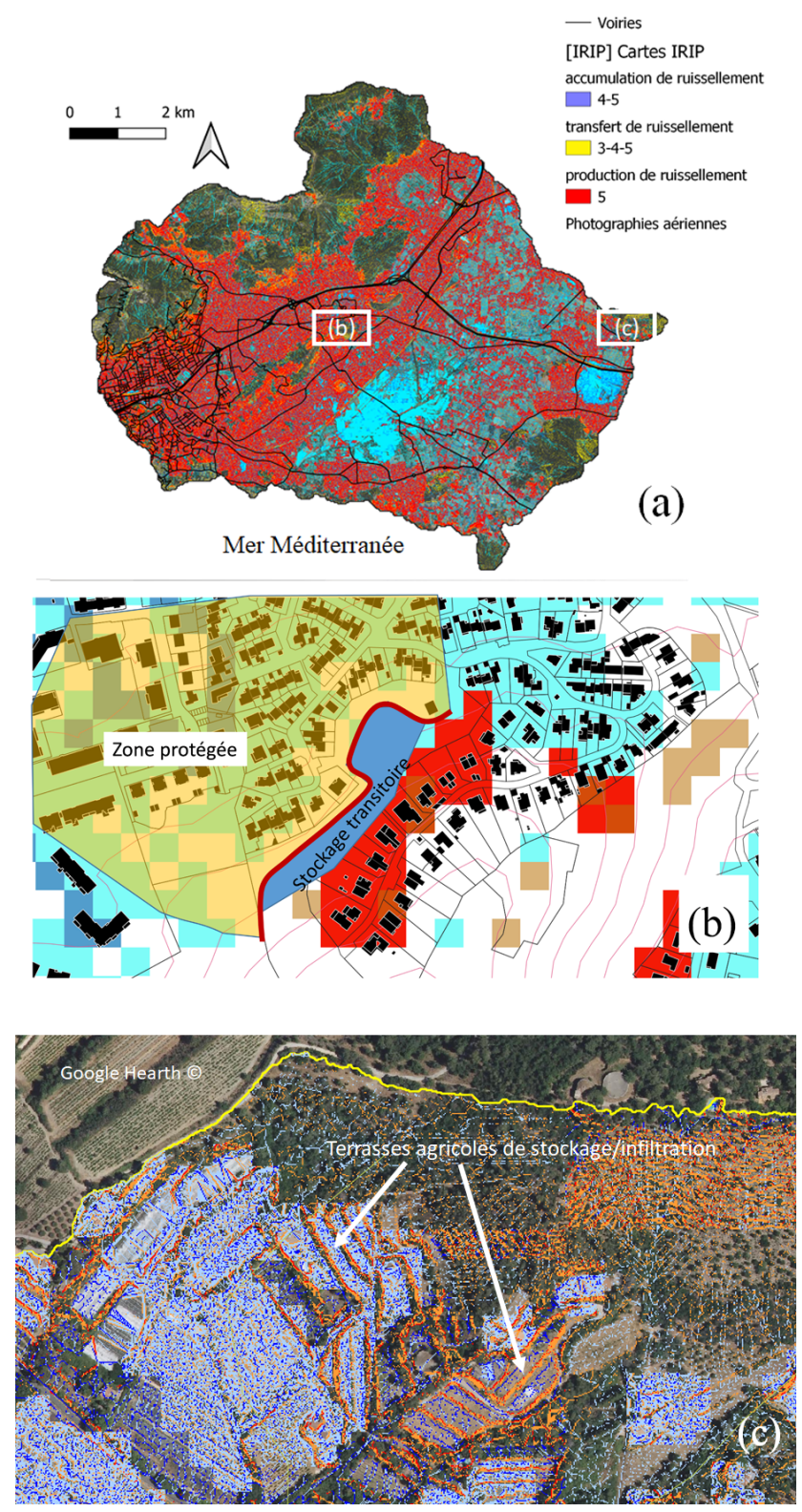

Figure 2. (a, b, c) Localisation des opportunités d'action pour réduire l'effet du ruissellement en périphérie de Toulon. 
ment pour évaluer la réduction des risques en aval et l'augmentation des services écosystémiques associés.

\section{Perspectives}

Dans les exemples traités, la cartographie du ruissellement intense ouvre des perspectives pour définir les zones d'intervention qui permettraient de réguler les flux d'eau et d'énergie en utilisant de manière équilibré les services écosystémiques. Ces services sont multiples, directs, indirects, visibles ou cachés. A titre d'exemple, les terrasses agricoles, « restanques en France ou fanya juu en Afrique de l'Est » réduisent l'érosion, le transfert des flux d'eaux et de substances, la propagation des incendies, et favorisent l'augmentation de l'infiltration, la rétention de la matière organique et la biodiversité. Une étude détaillée (Hammad et al., 2004) montre que la productivité agricole est pratiquement doublée sur les parcelles en terrasse en comparaison avec des parcelles en pente. La maintenance, voire même le développement de nouvelles terrasses agricoles dans les versants en pente modérée est une solution écohydrologique d'intérêt pour atténuer les effets négatifs du changement climatique et accroitre des services écosystémiques et aussi économiques. La recherche d'un équilibre écohydrologique dans un versant ou bassin versant implique une cohérence entre les dynamiques des processus naturels (temps de séjours nécessaires aux processus d'assimilation, de biotransformations) et l'usage des bénéfices (production agricole, autoépuration de l'eau) réalisés. Pour les milieux anthropisés cet équilibre repose dans un premier temps sur la réduction des facteurs de déséquilibre, comme les pertes en sol, l'usage de susbtances nocives pour les processus naturels de bio-transformation. Le modèle IRIP participe des outils qu'il convient de développer pour sectoriser l'usage des sols dans les zones en devenir d'urbanisation et pour conserver des fonctions écologiques en des points clés comme les zones d'accumulation. Ce modèle peut être utilisé en simulation de changement d'occupation du sol. Le changement de paradigme proposé ici consiste à limiter l'utilisation de solutions grises basées sur l'évacuation rapide des excès d'eau, comme cela a longtemps été pratiqué dans les pays développés. L'idée est au contraire de favoriser la rétention dynamique et la répartition des excès d'eau et de substances associées pour accroitre la productivité des services écosystémiques.

Le projet d'étude Dakar'2030 a été initié par les trois instituts cosignataires de cet article avec l'idée de réfléchir sur ce que pourrait devenir cette ville en 2030 si l'on pouvait penser autrement le développement urbain.

Disponibilité du code. Le modèle est un plugin pour QGIS3. Il est disponible librement auprès de l'animateur du projet Pascal Breil (pascal.breil@inrae.fr), avec un mode d'emploi et un jeu de données test en français et en anglais. Il utilise QGIS version 3 avec Grass, sous Windows, Linux et MAC-OS.
Disponibilité des données. Toutes les données utilisées sont accessibles gratuitement à partir des sources du Tableau 1. Il semble que le site basegeo du senegal soit actuellement hors d'usage.

Collaborateurs. Le modèle utilisé est développé par PB qui l'a appliqué aux deux cas d'étude présentés. Les données françaises ont été recueillies par PB, les données Sénégalaises par AF et DO. La rédaction résulte d'un travail collaboratif.

Intérêts concurrents. Les auteurs déclarent qu'ils n'ont aucun conflit d'intérêts.

Clause de non-responsabilité. Publisher's note : Copernicus Publications remains neutral with regard to jurisdictional claims in published maps and institutional affiliations.

Déclaration du numéro spécial. This article is part of the special issue "Hydrology of Large River Basins of Africa". It is a result of the 4th International Conference on the "Hydrology of the Great Rivers of Africa", Cotonou, Benin, 13-20 November 2021.

Remerciements. Les résultats présentés pour la ville de Toulon ont été financés par l'Agence de l'Eau Rhône Méditerranée Corse via l'action 65 de son accord cadre avec INRAE.

Financement. La participation à cette conférence et l'article associé ont bénéficié du soutien financier du département AQUA de l'INRAE dans le cadre de ses actions incitatives 2021.

\section{Références}

Braud, I., Lagadec, L.-R., Moulin, L., Chazelle, B., and Breil, P. : A method to use proxy data of runoff-related impacts for the evaluation of a model mapping intense storm runoff hazard : application to the railway context, Nat. Hazards Earth Syst. Sci., 20, 947-966, https://doi.org/10.5194/nhess-20-947-2020, 2020.

Bodian, A., Dacosta, H., Ndimag Diouf, R., Ndiaye, E. H. O., and Mendy, A. : Contribution à la connaissance de l'aléa pluvial au Sénégal grâce à la valorisation des données pluviographiques historiques, Journal de l'association internationale de Climatologie, 13, 38-46, accessible à l'adresse suivante : http://lodel. irevues.inist.fr/climatologie/index.php?id=1194 (la date du dernier accès : 20 mai 2021), 2016.

Cramer, W., Guiot, J., and Marini, K. : Les risques liés aux changements climatiques et environnementaux dans la région Méditerranée, MedECC, accessible à l'adresse suivante : https://ufmsecretariat.org/wp-content/uploads/2019/10/ MedECC-Booklet_FR_WEB.pdf (la date du dernier accès : 21 mai 2021), 2019.

Hammad, A. A., Haugen, L. E., and Borresen, T. : Effects of Stonewalled Terracing Techniques on Soil-Water Conservation 
and Wheat Production Under Mediterranean Conditions, Environ. Manage., 34, 701-710, https://doi.org/10.1007/s00267-0030278-9, 2004.

ISRIC data hub : Africa SoilGrids - Texture , ISRIC [data set], accessible à l'adresse suivante : https://data.isric.org/ geonetwork/srv/fre/catalog.search\#/home (la date du dernier accès : 21 mai 2021), 2020.

Lagadec, L.-R., Moulin, L., Braud, I., Chazelle, B., and Breil, P. : A surface runoff mapping method for optimizing risk analysis on railways, Safety Science, 110, 253-267, https://doi.org/10.1016/j.ssci.2018.05.014, 2018.
Zalewski, M. : Ecohydrology and Hydrologic Engineering : Regulation of Hydrology-Biota Interactions for Sustainability, J. Hydrol. Eng., 20, A4014012, https://doi.org/10.1061/(ASCE)HE.1943-5584.0000999, 2014. 